\title{
OUTPUT MANEUVERING FOR A CLASS OF NONLINEAR SYSTEMS
}

\author{
Roger Skjetne ${ }^{*, 1}$ Thor I. Fossen * Petar Kokotović ${ }^{* *}$ \\ * Department of Engineering Cybernetics, Norwegian \\ University of Science and Technology, N-7491 Trondheim, \\ Norway.E-mail: skjetne@ieee.org, tif@itk.ntnu.no \\ ** Center for Control Engineering and Computation, University \\ of California, Santa Barbara, CA 93106, USA. E-mail: \\ pk@ece.ucsb.edu
}

\begin{abstract}
The output maneuvering problem involves two tasks. The first, which is the geometric task, is to force the output to converge to a desired path parametrized by a continuous scalar variable $\theta$. The second task is to satisfy a desired speed assignment along the path. The main concern is to satisfy the geometric task. However, the speed assignment will ensure that the output follows the path with sufficient speed. A recursive control design technique is developed for nonlinear plants in vectorial strict feedback form of any relative degree. First the geometric part of the problem is solved. Then an update law is constructed that bridges the geometric design with the speed assignment. An extra degree of freedom is provided for an operator to specify the speed $\dot{\theta}$. A computer simulation with a marine vessel is performed to illustrate the design. Copyright (C)2002 IFAC
\end{abstract}

Keywords: Output maneuvering; Path following; Backstepping; Nonlinear control; Marine vessels.

\section{INTRODUCTION}

In many applications it is of primary importance to steer an object (ship,vehicle, robot arm, etc.) along a desired path. The speed assignment along the path may be less important. Vehicle control applications of this type are described in (Micaelli and Samson, 1993), (Hauser and Hindman, 1997) and (Pettersen and Lefeber, 2001), while an informative reference for robotic manufacturing plants is (Song et al., 2000).

Rather than considering a single tracking problem as a function of time, control problems for such applications are usually approached as two separate tasks. The first task is to reach and follow a desired path as a function of a scalar variable $\theta$, left as an extra degree of freedom for the second task. In the second task, $\theta$ is used to satisfy an additional specification of speed assignment.

\footnotetext{
1 Supported by the Norwegian Research Council through the Strategic University Program on Marine Cybernetics
}

Hauser and Hindman (1995) introduced a procedure to design a maneuver regulation controller which ensures that the states converge to the desired path and then proceed along the path. To determine the path variable $\theta$, they used a $P$-orthogonal projection from the current state onto the path. The already at hand tracking controller was then converted into a maneuver regulation controller, and a quadratic Lyapunov function was employed to guarantee that the states converge to the path and move along the path.

The methodology of Hauser and Hindman (1995) applies to feedback linearizable systems, where the desired path is specified for the full state. Encarnação and Pascoal (2001) further showed how the methodology of Hauser and Hindman (1995) can be extended to solve the output maneuvering problem by backstepping. However, for systems of relative degree higher than two, their approach has the disadvantage that each step of backstepping increases the number of time derivatives of $\theta$. 
The contribution of this paper is a maneuvering design that is applicable to systems in vectorial strict feedback form of any relative degree. In the $n$ recursive steps, the design solves the geometric part of the problem. It then proceeds to construct an update law that ties together the geometric design with the speed assignment. An extra degree of freedom is provided for an operator to select the speed of the maneuver variable $\theta$.

\subsection{An introductory example}

To introduce the main idea of the design procedure developed in Section 2, we look at the strict feedback form system

$$
\begin{aligned}
& \dot{x}_{1}=x_{2}+x_{1}^{2} \\
& \dot{x}_{2}=u
\end{aligned}
$$

where $y=x_{1}$ is the output and $u$ is the control. We want to design a controller to

\section{1. force the output $y$ to converge to the} path $y_{d}=\sin (\theta)$.

2. assign $\theta$ to $t-\phi$, that is, make the output $y$ to oscillate with frequency $1 \mathrm{rad} / \mathrm{s}$.

The design in Section 2 results in the control law

$$
\begin{array}{r}
u=-2\left[x_{1}+x_{1}^{2}+x_{1}^{3}+x_{1} x_{2}+x_{2}\right. \\
\left.-y_{d}(\theta)-y_{d}^{\prime}(\theta)-\frac{1}{2} y_{d}^{\prime \prime}(\theta)\right]
\end{array}
$$

where $y_{d}^{\prime}(\theta)=\frac{d y_{d}}{d \theta}(\theta)=\cos (\theta)$ and $y_{d}^{\prime \prime}(\theta)=$ $\frac{d^{2} y_{d}}{d \theta^{2}}(\theta)=-\sin (\theta)$. In the new coordinates

$$
\begin{aligned}
z_{1} & =x_{1}-y_{d}(\theta) \\
z_{2} & =x_{1}+x_{1}^{2}+x_{2}-y_{d}(\theta)-y_{d}^{\prime}(\theta) \\
\omega_{s} & =1-\dot{\theta}
\end{aligned}
$$

the resulting closed-loop system is

$$
\begin{aligned}
& \dot{z}_{1}=-z_{1}+z_{2}+y_{d}^{\prime}(\theta) \omega_{s} \\
& \dot{z}_{2}=-z_{1}-z_{2}+\left[y_{d}^{\prime}(\theta)+y_{d}^{\prime \prime}(\theta)\right] \omega_{s}
\end{aligned}
$$

For the Lyapunov function

$$
V=\frac{1}{2} z_{1}^{2}+\frac{1}{2} z_{2}^{2}+\frac{1}{2} \omega_{s}^{2}
$$

the derivative along the solutions of (6), (7) is

$\dot{V}=-z_{1}^{2}-z_{2}^{2}+\left\{z_{1} y_{d}^{\prime}(\theta)+z_{2}\left[y_{d}^{\prime}(\theta)+y_{d}^{\prime \prime}(\theta)\right]+\dot{\omega}_{s}\right\} \omega_{s}$

where an update law for $\dot{\omega}_{s}$ is yet to be assigned. To make $\dot{V}$ negative definite, we let

$$
\dot{\omega}_{s}=-\omega_{s}-z_{1} y_{d}^{\prime}(\theta)-z_{2}\left[y_{d}^{\prime}(\theta)+y_{d}^{\prime \prime}(\theta)\right]
$$

and achieve global exponential stability (GES) of the equilibrium $\left(z_{1}, z_{2}, \omega_{s}\right)=0$.

The main purpose of this example is to interpret how the design specifications have been met. From the GES property, it follows that $z_{1}(t)=y(t)-\sin (\theta(t)) \rightarrow 0$ and, hence, $y$ converges to $y_{d}=\sin (\theta)$. To recognize that the time assignment, $\theta(t) \rightarrow t-\phi$ has been met, we integrate $\dot{\theta}(t)=1-\omega_{s}(t)$ and get

$$
\theta(t)=t+\theta(0)-\int_{0}^{t} \omega_{s}(\tau) d \tau
$$

where $\phi=\lim _{t \rightarrow \infty}\left[\theta(0)-\int_{0}^{t} \omega_{s}(\tau) d \tau\right]$ exists because the convergence $\omega_{s}(t) \rightarrow 0$ is exponential. From (9) we see that $\theta$, and hence $y_{d}(\theta)$, depends on the system state. In this way, the desired transient to the path and the speed along the path, are shaped by feedback system dynamics. This flexibility is due to the fact that $\theta \rightarrow t-\phi$ is required to be attained only asymptotically, rather than as an identity $\theta=t-\phi$.

For another interpretation, we represent the closedloop system in the space of $\left(x_{1}, x_{2}, \theta, \omega_{s}\right)$ as follows

$$
\begin{aligned}
\dot{x}_{1}= & x_{2}+x_{1}^{2} \\
\dot{x}_{2}= & -2\left[x_{1}+x_{1}^{2}+x_{1}^{3}+x_{2}+x_{1} x_{2}\right] \\
& \quad+\sin (\theta)+2 \cos (\theta) \\
\dot{\theta}= & 1-\omega_{s} \\
\dot{\omega}_{s}= & -z_{1} \cos (\theta)-z_{2}[\cos (\theta)-\sin (\theta)]-\omega_{s}
\end{aligned}
$$

It can be verified that this dynamical system has an invariant manifold defined by

$$
\begin{aligned}
x_{1} & =\sin (\theta) \\
x_{2} & =\cos (\theta)-\sin ^{2}(\theta) \\
\omega_{s} & =0
\end{aligned}
$$

on which $x_{1}(t)$ and $\dot{x}_{1}(t)$ satisfy $x_{1}^{2}(t)+\dot{x}_{1}^{2}(t)=1$. Since this invariant manifold is globally attractive, $y(t)$ converges to the behavior of a harmonic oscillator as required by the design specifications. The phase $\phi$ of the oscillation is unspecified and depends on the initial condition.

A further design step can be used to assign the phase $\phi$, for example to $\phi=0$. Introducing $\omega_{t}=t-\theta$ such that

$$
\dot{\omega}_{t}=\omega_{s}
$$

and augmenting the Lyapunov function (8) by $\frac{1}{2} \omega_{t}^{2}$, a new update law for $\dot{\omega}_{s}$ is selected to be

$$
\dot{\omega}_{s}=-\omega_{s}-\omega_{t}-z_{1} y_{d}^{\prime}(\theta)-z_{2}\left[y_{d}^{\prime}(\theta)+y_{d}^{\prime \prime}(\theta)\right]
$$

Since the closed-loop (6), (7), (14), (15) becomes timevarying with $\theta=t-\omega_{t}(t)$, we resort to LaSalleYoshizawa (see Theorem 2.1 in Krstić et al. (1995)) to establish global boundedness of $\left(z_{1}, z_{2}, \omega_{t}, \omega_{s}\right)$ and convergence $\left(z_{1}, z_{2}, \omega_{s}\right) \rightarrow 0$. Using Theorem 1 in (Fossen et al., 2001), it is straightforward to show that the equilibrium $\left(z_{1}, z_{2}, \omega_{t}, \omega_{s}\right)=0$ is indeed UGAS. It follows that $\omega_{t}(t)=t-\theta(t) \rightarrow 0$ and, hence, $\phi(t) \rightarrow 0$.

\subsection{Definitions and problem statement}

Time derivatives of $x(t)$ are denoted $\dot{x}, \ddot{x}, x^{(3)}, \ldots, x^{(i)}$, and derivatives with respect to $\theta$ 
of $x(\theta)$ are $x^{\prime}, x^{\prime \prime}, x^{\left(3^{\prime}\right)}, \ldots, x^{\left(i^{\prime}\right)}$. We denote $x^{(1 \ldots i)}$ [or $x^{\left(1^{\prime} \ldots i^{\prime}\right)}$ ] as a collection of derivatives of $x(t)$ [or $x(\theta)]$.

$\bar{x}_{i}$ denotes the vector $\bar{x}_{i}=\left[\begin{array}{llll}x_{1}^{\top} & x_{2}^{\top} & \ldots & x_{i}^{\top}\end{array}\right]^{\top}$.

A Parametrized Path is a geometric curve $\xi \in \Re^{q}$, $q \geq 1$, that is parametrized by a continuous variable $\theta$.

The Path Characterization Vector is the vector

$\xi_{c v}(\theta) \triangleq\left[\left(\xi^{\prime}(\theta)\right)^{\top},\left(\xi^{\prime \prime}(\theta)\right)^{\top}, \ldots,\left(\xi^{\left(n^{\prime}\right)}(\theta)\right)^{\top}\right]^{\top}$

The Maneuvering Problem: design a controller that solves the two tasks:

1. The Geometric Task: force the state $x$ to converge to a desired path $\xi(\theta)$,

$$
\lim _{t \rightarrow \infty}[x(t)-\xi(\theta(t))]=0
$$

for any continuous function $\theta(t)$.

2. The Speed Assignment Task: force the speed $\dot{\theta}$ to converge to a desired speed $v_{s}$,

$$
\lim _{t \rightarrow \infty}\left[\dot{\theta}(t)-v_{s}(\theta, t)\right]=0
$$

The Output Maneuvering Problem is to solve the maneuvering problem with respect to the output $y$ rather than the state $x$.

A more restrictive version of the asymptotic speed assignment (17) is to satisfy it identically, $\dot{\theta} \equiv v_{s}$. In particular, if $v_{s}=1$, then the maneuvering problem becomes a tracking problem where the desired output $y_{d}(\theta(t))$ will be a prespecified time signal $\tilde{y}_{d}(t)$. Using the asymptotic formulation (17), on the other hand, allows for more flexibility, including the possibility to let $\dot{\theta}$ depend on the system state.

\section{OUTPUT MANEUVERING}

Consider the general nonlinear system in strict feedback form with vector relative degree $n$

$$
\begin{aligned}
\dot{x}_{1}= & G_{1}\left(\bar{x}_{1}\right) x_{2}+f_{1}\left(\bar{x}_{1}\right) \\
\dot{x}_{2}= & G_{2}\left(\bar{x}_{2}\right) x_{3}+f_{2}\left(\bar{x}_{2}\right) \\
& \vdots \\
\dot{x}_{n}= & G_{n}\left(\bar{x}_{n}\right) u+f_{n}\left(\bar{x}_{n}\right) \\
y= & h\left(x_{1}\right)
\end{aligned}
$$

where $x_{i} \in \Re^{m}, \quad i=1, \ldots, n, y \in \Re^{m}$ is the output and $u \in \Re^{m}$ is the control. The state dependent matrices $G_{i}\left(\bar{x}_{i}\right)$ and $\frac{\partial h}{\partial x_{1}}\left(x_{1}\right)$ are invertible for all $\bar{x}_{i}$, and $G_{i}, f_{i}$ and $h$ are smooth.

The control objective is to design a maneuvering controller that solves the output maneuvering problem with respect to a desired parametrized output path

$$
\mathbf{Y}_{d}=\left\{y_{d}(\theta) \mid y_{d} \in \Re^{m}, \theta \in \Re\right\}
$$

where $y_{d}$ is $n$ times differentiable with respect to $\theta$.

\subsection{Design procedure}

In the recursive procedure that follows, the first two steps are separated from steps 3 through $n$ since the first two steps involve $\frac{\partial h}{\partial x_{1}}$. The design procedure borrows much from adaptive tracking developed by Krstic et al. (1995), and the notion of a tuning function is used also in this design.

\section{Step 1:}

The new variables

$$
\begin{aligned}
& \omega_{s} \triangleq v_{s}-\dot{\theta} \\
& z_{1} \triangleq y-y_{d}(\theta) \\
& z_{i} \triangleq x_{i}-\alpha_{i-1}, \quad i=2, \ldots, n
\end{aligned}
$$

are introduced, where $v_{s}$ is a bounded $\mathcal{C}^{n-1}$ signal, and $\alpha_{i-1}$ are virtual controls to be specified later. Differentiating (21) with respect to $t$ results in

$$
\begin{aligned}
\dot{z}_{1}= & \dot{y}-y_{d}^{\prime} \dot{\theta} \\
= & \left(\frac{\partial h}{\partial x_{1}}\left(x_{1}\right)\right) G_{1}\left(x_{1}\right) z_{2}+\left(\nabla h\left(x_{1}\right)\right) G_{1}\left(x_{1}\right) \alpha_{1} \\
& +\left(\frac{\partial h}{\partial x_{1}}\left(x_{1}\right)\right) f_{1}\left(x_{1}\right)-\nu_{1} v_{s}+\nu_{1} \omega_{s}
\end{aligned}
$$

where $\nu_{1} \triangleq y_{d}^{\prime}$. Using $p_{1}>0$, define the first Lyapunov function as

$$
V_{1} \triangleq p_{1} z_{1}^{\top} z_{1}
$$

whose time derivative is

$$
\begin{aligned}
\dot{V}_{1}= & 2 p_{1} z_{1}^{\top}\left[\left(\frac{\partial h}{\partial x_{1}}\right) G_{1} \alpha_{1}+(\nabla h) f_{1}-\nu_{1} v_{s}\right] \\
& +2 p_{1} z_{1}^{\top}\left(\frac{\partial h}{\partial x_{1}}\right) G_{1} z_{2}+2 p_{1} z_{1}^{\top} \nu_{1} \omega_{s}
\end{aligned}
$$

Then the first virtual control is picked as

$$
\begin{aligned}
\alpha_{1} & =G_{1}^{-1}\left(\frac{\partial h}{\partial x_{1}}\right)^{-1}\left[-C_{1} z_{1}-(\nabla h) f_{1}+\nu_{1} v_{s}\right] \\
& =f_{\alpha_{1}}\left(x_{1}, y_{d}, y_{d}^{\prime}, v_{s}\right)
\end{aligned}
$$

where $C_{1}=C_{1}^{\top}>0$. Let $Q_{1} \triangleq 2 p_{1} C_{1}$ and define the first tuning function, $\tau_{1} \in \Re$, as

$$
\tau_{1} \triangleq 2 p_{1} z_{1}^{\top} \nu_{1}
$$

Finalizing Step 1, the result is

$$
\begin{aligned}
& \dot{V}_{1}=-z_{1}^{\top} Q_{1} z_{1}+2 p_{1} z_{1}^{\top}\left(\frac{\partial h}{\partial x_{1}}\right) G_{1} z_{2}+\tau_{1} \omega_{s} \\
& \dot{z}_{1}=-C_{1} z_{1}+\left(\frac{\partial h}{\partial x_{1}}\right) G_{1} z_{2}+\nu_{1} \omega_{s}
\end{aligned}
$$

where $z_{2}$ and $\omega_{s}$ are left for the next step. In aid of next step, let

$$
\dot{\alpha}_{1}=\sigma_{1}+\nu_{2} \dot{\theta}
$$

where $\sigma_{1}$ is defined as the terms in $\dot{\alpha}_{1}$ not containing $\dot{\theta}$, and $\nu_{2}$ as those multiplying $\dot{\theta}$, that is

$$
\begin{gathered}
\sigma_{1} \triangleq \frac{\partial \alpha_{1}}{\partial x_{1}} \dot{x}_{1}+\frac{\partial \alpha_{1}}{\partial v_{s}} \dot{v}_{s} \\
\nu_{2} \triangleq \frac{\partial \alpha_{1}}{\partial y_{d}} y_{d}^{\prime}+\frac{\partial \alpha_{1}}{\partial y_{d}^{\prime}} y_{d}^{\prime \prime}
\end{gathered}
$$

Steps 2 and $i=3, \ldots, n$ are summarized in Tables 1 and 2 . 


$$
\dot{z}_{n}=-\frac{p_{n-1}}{p_{n}} G_{n-1}^{\top} z_{n-1}-C_{n} z_{n}+\nu_{n} \omega_{s}
$$

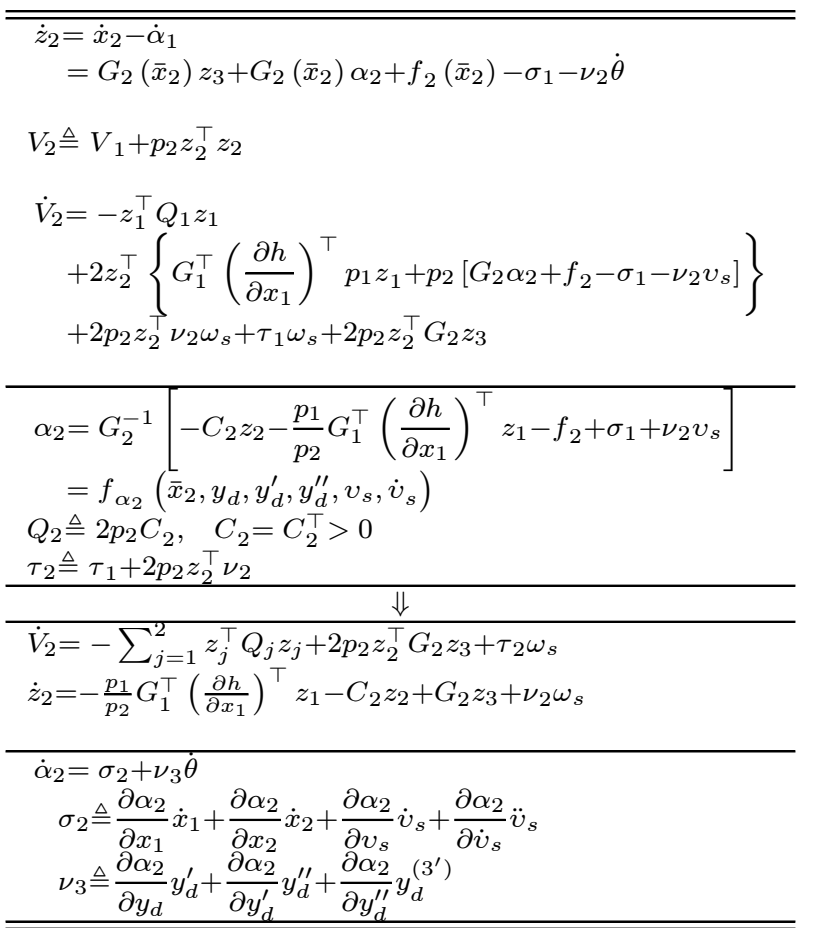

Table 2. Step $\mathbf{i}=\mathbf{3}, \ldots, \mathbf{n}$

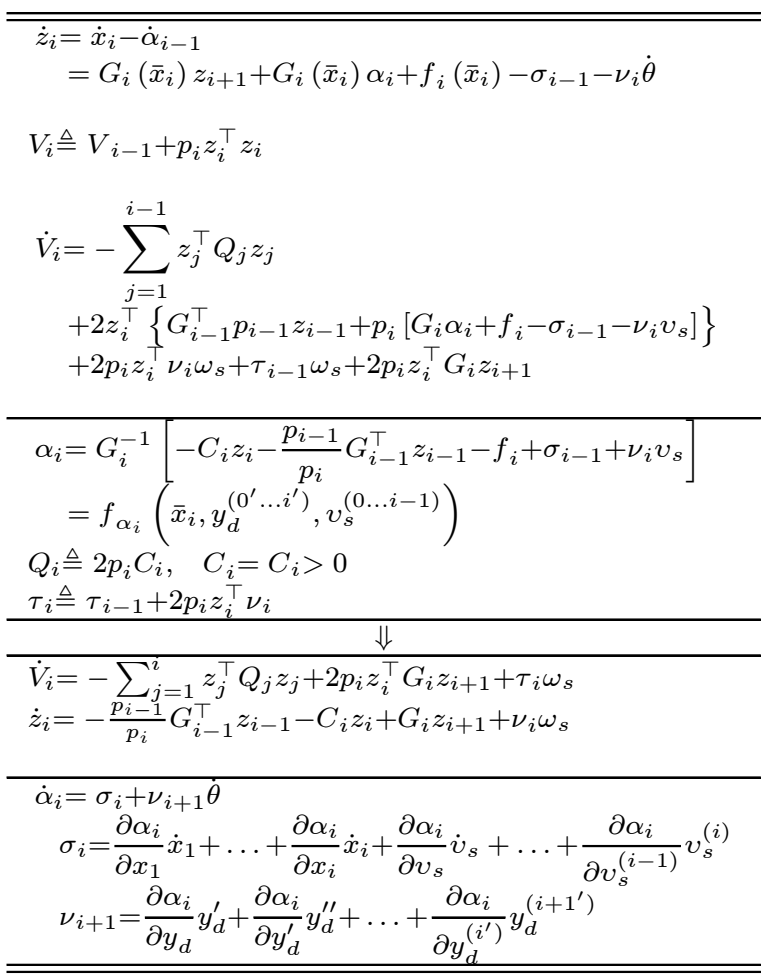

Upon the completion of Step $n$ the choice of the control law is

$$
\begin{aligned}
u= & \alpha_{n} \\
=G_{n}^{-1}[- & C_{n} z_{n}-\frac{p_{n-1}}{p_{n}} G_{n-1}^{\top} z_{n-1} \\
& \left.\quad-f_{n}+\sigma_{n-1}+\nu_{n} v_{s}\right]
\end{aligned}
$$

results in

$$
\dot{V}_{n}=-\sum_{j=1}^{n} z_{j}^{\top} Q_{j} z_{j}+\tau_{n} \omega_{s}
$$

This implies that, if $\omega_{s}=0$, then $z=\left[z_{1}^{\top}, \ldots, z_{n}^{\top}\right]^{\top}$ will converge to $z=0$ as $t \rightarrow \infty$. Hence, $y(t) \rightarrow$ $y_{d}(\theta(t))$ for any continuous $\theta(t)$, and the geometric task (16) is solved.

\subsection{Speed assignment}

To solve the speed assignment task (17), augment the Step $n$ Lyapunov function to

$$
V=V_{n}+\frac{1}{2 \mu_{1}} \omega_{s}^{2}, \quad \mu_{1}>0
$$

The time derivative of (32) is

$$
\begin{aligned}
\dot{V} & =\dot{V}_{n}+\frac{1}{\mu_{1}} \omega_{s} \dot{\omega}_{s} \\
& =-\sum_{j=1}^{n} z_{j}^{\top} Q_{j} z_{j}+\left[\tau_{n}+\frac{1}{\mu_{1}} \dot{\omega}_{s}\right] \omega_{s}
\end{aligned}
$$

where the update law for $\dot{\omega}_{s}$ is yet to be constructed. To make $\dot{V}$ negative definite, choose the $\dot{\omega}_{s}$-update law

$$
\dot{\omega}_{s}=-\lambda \omega_{s}-\mu_{1} \tau_{n}
$$

$\lambda>0$, which gives

$$
\dot{V}=-\sum_{j=1}^{n} z_{j}^{\top} Q_{j} z_{j}-\frac{\lambda}{\mu_{1}} \omega_{s}^{2}<0
$$

Consequently, as $t \rightarrow \infty, \omega_{s}(t)=v_{s}(\theta, t)-\dot{\theta}(t) \rightarrow$ 0 , and the speed assignment (17) is satisfied. From (33) it follows that $\omega_{s}(t)$, and therefore $y_{d}(\theta(t))$, is dependent on the system state $x(t)$ through the final tuning function $\tau_{n}$. Hence, feedback is introduced from the system state to the desired output. This feedback gives the overall system certain good properties compared to a pure tracking design.

\subsection{The main result}

To obtain the closed-loop system in vector form, let $A_{z}\left(\bar{x}_{n-1}\right)$ and $F\left(\bar{x}_{n}, v_{s}^{(0 \ldots n-2)}(\theta, t)\right)$ be as below

$$
\begin{aligned}
& A_{z}\left(\bar{x}_{n-1}\right)=
\end{aligned}
$$

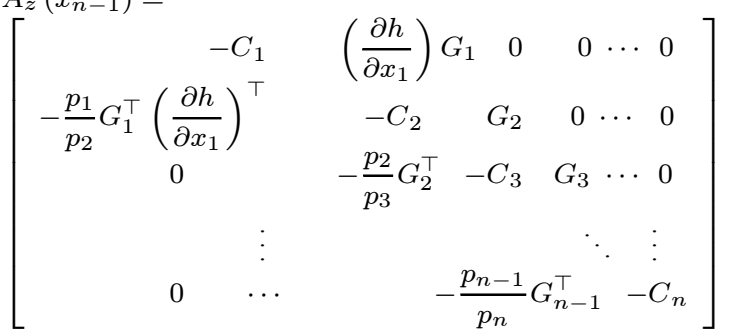

$$
\begin{aligned}
& \begin{array}{l}
F\left(\bar{x}_{n}, v_{s}^{(0 \ldots n-2)}(\theta, t)\right)= \\
{\left[\begin{array}{ccccc}
I & 0 & 0 & \cdots & 0 \\
\frac{\partial \alpha_{1}}{\partial y_{d}}(t) & \frac{\partial \alpha_{1}}{\partial y_{d}^{\prime}}(t) & 0 & \cdots & 0 \\
\frac{\partial \alpha_{2}}{\partial y_{d}}(t) & \frac{\partial \alpha_{2}}{\partial y_{d}^{\prime}}(t) & \frac{\partial \alpha_{2}}{\partial y_{d}^{\prime \prime}}(t) & \cdots & 0 \\
\vdots & \vdots & \vdots & \ddots & \vdots \\
\frac{\partial \alpha_{n-1}}{\partial y_{d}}(t) & \frac{\partial \alpha_{n-1}}{\partial y_{d}^{\prime}}(t) & \frac{\partial \alpha_{n-1}}{\partial y_{d}^{\prime \prime}}(t) & \cdots & \frac{\partial \alpha_{n-1}}{\partial y_{d}^{\left(n-1^{\prime}\right)}}(t)
\end{array}\right]}
\end{array}
\end{aligned}
$$


Define the path characterization vector as

$\xi_{c v}(\theta) \triangleq\left[\left(y_{d}^{\prime}(\theta)\right)^{\top}\left(y_{d}^{\prime \prime}(\theta)\right)^{\top} \ldots\left(y_{d}^{\left(n^{\prime}\right)}(\theta)\right)^{\top}\right]^{\top}$

which gives the relationship

$$
\tau_{n}=2 \xi_{c v}^{\top}(\theta) F^{\top}\left(\bar{x}_{n}, v_{s}^{(0 \ldots n-2)}(\theta, t)\right) P z .
$$

with $z=\left[z_{1}^{\top}, \ldots, z_{n}^{\top}\right]^{\top}$ and $P \triangleq \operatorname{diag}\left(p_{1} I, p_{2} I, \ldots\right.$, $\left.p_{n} I\right)$. In vector form, the closed-loop equation is

$$
\begin{gathered}
\dot{z}=A_{z}\left(\bar{x}_{n-1}\right) z+F\left(\bar{x}_{n}, v_{s}^{(0 \ldots n-2)}\right) \xi_{c v}(\theta) \omega_{s} \\
\dot{\omega}_{s}=-2 \mu_{1} \xi_{c v}^{\top}(\theta) F^{\top}\left(\bar{x}_{n}, v_{s}^{(0 \ldots n-2)}\right) P z-\lambda \omega_{s}
\end{gathered}
$$

Under the following assumption

(A1) There exist a positive real, $r_{1}>0$, such that the path characterization vector $\xi_{c v}(\theta)$ is upper bounded, uniformly in $\theta$, that is,

$$
0 \leq\left|\xi_{c v}(\theta)\right|<r_{1}<\infty, \quad \forall \theta \in \Re
$$

we have the result:

Theorem 1. The closed-loop output maneuvering system consisting of the plant (18), the control (29) and the $\omega_{s}$-update law (33), where $\omega_{s}$ is given by (20), has a Uniformly Globally Exponentially Stable (UGES) equilibrium $\left(z, \omega_{s}\right)=(0,0)$. This means in particular that global exponential output maneuvering of the output path (19) is achieved:

$$
\begin{aligned}
\lim _{t \rightarrow \infty}\left[y(t)-y_{d}(\theta(t))\right] & =0 \\
\lim _{t \rightarrow \infty}\left[\dot{\theta}(t)-v_{s}(\theta(t), t)\right] & =0
\end{aligned}
$$

Remark 1. In the case of constant $v_{s}(\theta, t)=v_{0} \neq 0$, all derivatives $v_{s}^{(i)}=0, i \geq 1$, and the closed-loop system, (35) and (36), is time-invariant. It follows that $\lim _{t \rightarrow \infty} \theta(t)=v_{0} t-\phi$, where

$\phi=\lim _{t \rightarrow \infty}\left[\theta(0)-\int_{0}^{t} \omega_{s}(\tau) d \tau\right]$ exists because the convergence $\omega_{s}(t) \rightarrow 0$ is exponential.

\subsection{Phase assignment}

For the constant $v_{s}(\theta, t)=v_{0} \neq 0$ case, it is possible to introduce a design step to assign the phase $\phi$. Let $\omega_{t}=v_{0} t-\theta-\phi_{0}$ where $\phi_{0}$ is a desired constant phase shift, for example $\phi_{0}=0$. The time derivative is

$$
\dot{\omega}_{t}=\omega_{s}
$$

and augmenting the Lyapunov function (32), to

$$
V=V_{n}+\frac{\mu_{0}}{2} \omega_{t}^{2}+\frac{1}{2 \mu_{1}} \omega_{s}^{2}, \quad \mu_{0} \geq 0
$$

a new update law for $\dot{\omega}_{s}$ is constructed as

$$
\dot{\omega}_{s}=-\lambda \omega_{s}-\mu_{1}\left[\tau_{n}+\mu_{0} \omega_{t}\right]
$$

This gives the negative semi-definite derivative of (38) along the solutions of (35), (37) and (39)

$$
\dot{V}=-z^{\top} Q z-\frac{\lambda}{\mu_{1}} \omega_{s}^{2} \leq 0
$$

Note that $\mu_{0}=0$ is acceptable in order to switch off the regulation of the phase. Under the assumptions

(A2) The path $y_{d}(\theta)$ is uniformly bounded in $\theta$.

(A3) The map $h\left(x_{1}\right)$ is a local diffeomorphism.

we have the following result:

Theorem 2. The closed-loop output maneuvering system consisting of the plant (18), the control (29), the update law (39) and (37), with $v_{s}(\theta, t)=v_{0} \neq 0$, has a Uniformly Globally Asymptotically Stable (UGAS) equilibrium $\left(z, \omega_{t}, \omega_{s}\right)=(0,0,0)$. In particular, this means that global asymptotic maneuvering of (19) is achieved:

$$
\begin{aligned}
\lim _{t \rightarrow \infty}\left[y(t)-y_{d}(\theta(t))\right] & =0 \\
\lim _{t \rightarrow \infty}\left[\dot{\theta}(t)-v_{0}\right] & =0
\end{aligned}
$$

In addition, any constant phase specification $\phi_{0}$ is satisfied, that is

$$
\left.\lim _{t \rightarrow \infty}\left[\theta(t)-v_{0} t+\phi_{0}\right)\right]=0
$$

Proof. First, notice that with $v_{s}(\theta, t)=v_{0}$ and $\theta(t)=$ $v_{0} t-\omega_{t}(t)-\phi_{0}$, the closed-loop equations (35), (39) and (37) are time-varying. From assumption A3 and the smooth virtual controls $\alpha_{i}$, there exist a continuous map $\Psi:(z, t) \rightarrow x$ which is uniformly bounded since $z(t)$ is exponentially converging and the time-variation only enters through either $y_{d}(\theta(t))$ or $\xi_{c v}(\theta(t))$, which due to assumptions A1 and A2 are uniformly bounded. Resorting to Theorem 1 in (Fossen et al., 2001) where $x_{1}=\operatorname{col}\left(z, \omega_{s}\right)$ and $x_{2}=\omega_{t}$, it can be verified that all assumptions are satisfied and that, therefore, the equilibrium $\left(z, \omega_{t}, \omega_{s}\right)=(0,0,0)$ is UGAS. It follows that as $t \rightarrow \infty, z_{1}=y(t)-y_{d}(\theta(t)) \rightarrow 0, \omega_{s}(t)=$ $v_{0}-\dot{\theta}(t) \rightarrow 0$ and $\omega_{t}(t)=v_{0} t-\theta(t)-\phi_{0} \rightarrow 0$.

\section{APPLICATION TO SHIP MANEUVERING}

For ship maneuvering, a dynamical model where the surge mode is decoupled from the sway and yaw modes is used. See Skjetne and Fossen (2001) and references therein. Let $\eta=\left[\begin{array}{lll}x & y & \psi\end{array}\right]^{\top}$ be the earth-fixed position vector and $\nu=\left[\begin{array}{ll}u & v\end{array}\right]^{\top}$ be the body-fixed velocity vector. The kinematics and dynamics is given by

$$
\begin{aligned}
& \dot{\eta}=R(\psi) \nu \\
& \dot{\nu}=M^{-1} N(\nu) \nu+M^{-1} G T
\end{aligned}
$$

where $M=M^{\top}>0$ and $T=\left[T_{u}, \delta_{1}, \delta_{2}\right]^{\top}$ is the control vector. The dynamical system (41) and (42) are then in the form of (18) where $\eta$ is the output and $T$ is the control. Let a desired output path be

$$
\eta_{d}(\theta)=\left[x_{d}(\theta) y_{d}(\theta) \arctan \left(\frac{y_{d}^{\prime}}{x_{d}^{\prime}}\right)\right]^{\top}
$$

where $x_{d}(\theta)$ and $y_{d}(\theta)$ are three times differentiable with respect to $\theta$. The control objective is:

1. force the ship to converge to and follow the path.

2. let the ship speed be adjustable online. 
Using the design procedure of Section 2, we ensure convergence to the path by forcing the convergence of the error state, $z_{1}(t) \rightarrow 0$. To solve the problem of online adjustable speed, let $u_{0}$ be a constant desired set-point for $u$. We then have the feedforward terms

$$
\begin{aligned}
v_{s}(\theta) & =\frac{u_{0}}{\sqrt{\left(x_{d}^{\prime}(\theta)\right)^{2}+\left(y_{d}^{\prime}(\theta)\right)^{2}}} \\
\dot{v}_{s} & =\frac{-\left[x_{d}^{\prime}(\theta) x_{d}^{\prime \prime}(\theta)+y_{d}^{\prime}(\theta) y_{d}^{\prime \prime}(\theta)\right] \dot{\theta}}{\left[\left(x_{d}^{\prime}(\theta)\right)^{2}+\left(y_{d}^{\prime}(\theta)\right)^{2}\right]^{3 / 2}} u_{0}
\end{aligned}
$$

The design procedure of Section 2 gives the following signals

$$
\begin{aligned}
z_{1} & =\eta-\eta_{d}(\theta) \\
z_{2} & =\nu-\alpha_{1}\left(\eta, \eta_{d}(\theta), \eta_{d}^{\prime}(\theta), v_{s}(\theta)\right) \\
\alpha_{1} & =R^{\top}(\psi)\left[-K_{p} z_{1}+\eta_{d}^{\prime}(\theta) v_{s}(\theta)\right] \\
\sigma_{1} & =\dot{R}^{\top}(\psi) R(\psi) \alpha_{1}+R^{\top}(\psi)\left[-K_{p} R(\psi) \nu+\eta_{d}^{\prime}(\theta) \dot{v}_{s}\right] \\
\nu_{2} & =R^{\top}(\psi)\left[K_{p} \eta_{d}^{\prime}(\theta)+\eta_{d}^{\prime \prime}(\theta) v_{s}(\theta)\right]
\end{aligned}
$$

The control law is given by

$$
\begin{array}{r}
T=G^{-1} M\left[-K_{d} z_{2}-P_{2}^{-1} R^{\top}(\psi) P_{1} z_{1}\right. \\
\left.-M^{-1} N \nu+\sigma_{1}+\nu_{2} v_{s}(\theta)\right]
\end{array}
$$

and the update law for $\dot{\omega}_{s}$ is

$$
\dot{\omega}_{s}=-\lambda \omega_{s}-2 \mu_{1}\left[z_{1}^{\top} P_{1} \eta_{d}^{\prime}(\theta)+z_{2}^{\top} P_{2} \nu_{2}\right]
$$

The simulation are done for a container ship of length $L=175 \mathrm{~m}$, and the numerical values are taken from Appendix E.1.3 in (Fossen, 1994). The desired output path are given by (43) where $x_{d}(\theta)=\theta$ and $y_{d}(\theta)=$ $500 \sin \left(\frac{2 \pi}{4000} \theta\right)$. The controller settings are as follows: $K_{p}=\operatorname{diag}(0.1,0.25,0.25), K_{d}=\operatorname{diag}(0.4,0.02$, $0.02), P_{1}=\operatorname{diag}(0.001,0.01,0.01), P_{2}=\operatorname{diag}(5$, 20, 20), $\lambda=0.1$ and $\mu_{1}=0.005$. Initial conditions are $\eta(0)=\left[400,0, \frac{\pi}{2}\right]^{\top}, \nu(0)=[4,0,0]^{\top}, \theta(0)=0$ and $\omega_{s}(0)=-1$. In Figure (1) it is shown how the ship accurately converges to and follows the path.

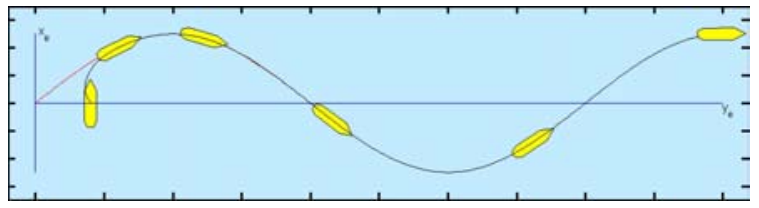

Fig. 1. Simulation of a container ship following a desired sinusoidal path on the ocean surface.

In Figure (2) it is shown how the surge speed $u$, by the command of an operator, is first controlled to $u_{0}=$ $4 \mathrm{~m} / \mathrm{s}$ and then at $t=400 \mathrm{~s}$ to $u_{0}=10 \mathrm{~m} / \mathrm{s}$.

\section{CONCLUSION}

As defined in section one, the maneuvering problem is separated into two tasks, the geometric task and the speed assignment task. The recursive design procedure developed in this paper shows one solution to the maneuvering problem for nonlinear systems in vectorial strict feedback form. In $n$ recursive steps, the geometric task was solved. Finally, an update law

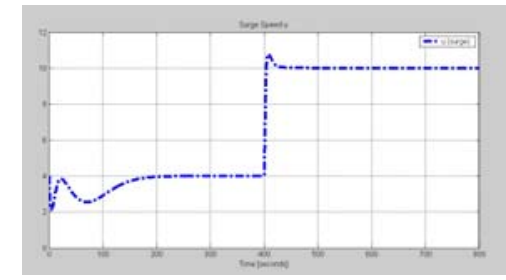

Fig. 2. Plot of the surge speed $u$ in the container ship simulation.

was constructed to satisfy the speed assignment as an asymptotic limit rather than as an identity. This update law introduced feedback from the system state to the desired output. In the case of constant desired speed, $\dot{\theta}=v_{0}$, a further design step was introduced to assign a constant desired phase $\phi=\phi_{0}$.

\section{REFERENCES}

Encarnação, P. and A. Pascoal (2001). Combined trajectory tracking and path following for marine craft.. In: Proc. 9th Mediterranean Conf. on Control and Automation. Dubrovnik, Croatia.

Fossen, T.I. (1994). Guidance and Control of Ocean Vehicles. John Wiley \& Sons Ltd.. England.

Fossen, T.I., A. Loría and A.R. Teel (2001). A theorem for UGAS and ULES of (passive) nonautonomous systems: robust control of mechanical systems and ships. Int. J. Robust Nonlinear Control 11, 95-108.

Hauser, J. and R. Hindman (1995). Maneuvering regulation from trajectory tracking: Feedback linearizable systems. In: Proc. IFAC Symposium on Nonlinear Control Systems Design. IFAC. Lake Tahoe, CA, USA. pp. 595-600.

Hauser, J. and R. Hindman (1997). Aggressive flight maneuvers. In: Proc. 36th IEEE Conf. Decision \& Control. IEEE. San Diego, California, USA. pp. 4186-4191.

Krstić, M., I. Kanellakopoulos and P.V. Kokotović (1995). Nonlinear and Adaptive Control Design. John Wiley \& Sons Ltd.. New York.

Micaelli, A. and C. Samson (1993). Trajectory tracking for unicycle-type and two-steering-wheels mobile robots.. Research Report 2097. Inst. National de Recherche en Informatique et en $\mathrm{Au}-$ tomatique.

Pettersen, K.Y. and E. Lefeber (2001). Way-point tracking control of ships.. In: Proc. 40th IEEE Conf. Decision \& Control. IEEE. To appear.

Skjetne, R. and T.I. Fossen (2001). Nonlinear maneuvering and control of ships. In: Proc. MTS/IEEE OCEANS 2001. IEEE. Honolulu, Hawaii. pp. 1808-1815.

Song, M., T.-J. Tarn and N. Xi (2000). Integration of task scheduling, action planning, and control in robotic manufacturing systems. In: Proc. IEEE Manuf. Syst. Vol. 88. IEEE. Ford Motor Co., Dearborn, MI, USA. pp. 1097-1107. 